\title{
DERIVATIONS AND (HYPER)INVARIANT SUBSPACES OF A BOUNDED OPERATOR
}

\author{
SHUANG ZHANG
}

(Communicated by John B. Conway)

\begin{abstract}
Let $X$ be a complex Banach space and $\mathcal{L}(X)$ the set of bounded linear operators on $X$. For $T \in \mathcal{L}(X)$, a derivation $\Delta_{T}$ is defined by $\Delta_{T} A=$ $T A-A T$ for $A \in \mathcal{L}(X)$. By induction, $\Delta_{T}^{m}=\Delta_{T} \circ \Delta_{T}^{m-1}$ is defined for each integer $m \geq 2$. We call the kernel of $\Delta_{T}^{m}$ the $m$-commutant of $T$. For a polynomially compact operator $T$, we consider the (hyper)invariant subspace problem for operators in the $m$-commutant of $T$ for $m \geq 1$. It is easily seen that the $m$-commutant $(m>1)$ of $T$ could be much larger than $\operatorname{Ker}\left(\Delta_{T}\right)$. So our idea is a variation of Lomonosov's theorem in [6]. We start with several identities on derivations, and then prove our results on the existence of (hyper)invariant subspaces. Theorem 2 in [5] is generalized.
\end{abstract}

In this paper, we always assume that $\operatorname{dim} X=\infty$. For a bounded operator $T$ on $X$ and a complex number $\alpha$, we denote by $X_{T}(\alpha)$ the norm closure of the linear manifold $\bigvee_{n=1}^{\infty} \operatorname{ker}(T-\alpha)^{n}$. If $A$ is a nonscalar bounded operator on $X$, we say that Lat $A$ is nontrivial if $A$ has a nontrivial (closed) invariant subspace. Similarly we say that $H$-Lat $A$ is nontrivial if $A$ has a nontrivial (closed) hyperinvariant subspace. For the sake of brevity, we state our main results in one theorem as follows.

THEOREM. If $T \in \mathcal{L}(X)$ is a polynomially compact operator with minimal polynomial $p(z)=\prod_{i=1}^{k}\left(z-\alpha_{i}\right)^{n_{i}}\left(n_{i} \geq 1\right.$ for each $i, k \geq 1 ; \alpha_{i} \neq \alpha_{j}$ if $\left.1 \leq i<j \leq k\right)$, and if $A$ is a nonscalar bounded operator on $X$ which is in the $(m+1)$-commutant of $T$ for some $m \geq 0$, then we have the following conclusions:

(a) if $T$ is algebraic and $\sigma(T)$ has at least two elements, then Lat $A$ is nontrivial;

(b) if $\sigma(p(T)) \neq\{0\}$, then $H$-Lat $A$ is nontrivial;

(c) if $\sigma(p(T))=\{0\}, k>1$, and $0<\operatorname{rank} \Delta_{T}^{m} A<\infty$, then Lat $A$ is nontrivial; For the special cases $m \leq 1$, we have

(d) if $T$ is not algebraic and $\operatorname{rank} \Delta_{T} A<\infty$, then $H$-Lat $A$ is nontrivial;

(e) if $\operatorname{rank} \Delta_{T} A=n_{0}<\infty$ and $q(T)=\left[\prod_{i=1}^{k}\left(T-\alpha_{i}\right)\right]^{m_{0}} \neq 0$, where $m_{0}=$ $\max \left\{n_{0}, n_{1}, \ldots, n_{k}\right\}$, then $H$-Lat $A$ is nontrivial.

Because the proof of the theorem is long, we divide it into several steps. We begin with some algebraic identities.

IDENTITY I.

$$
\Delta_{T^{n}} A^{m}=\sum_{i=0}^{n-1} \sum_{j=0}^{m-1} T^{n-i-1} A^{m-j-1}\left(\Delta_{T} A\right) A^{j} T^{i}
$$

for any integer $n \geq 1$ and $m \geq 1$.

Received by the editors March 15, 1986 and, in revised form, July 3, 1986 and September 11, 1986.

1980 Mathematics Subject Classification (1985 Revision). Primary 47A15, 47 B47.

Key words and phrases. Invariant subspaces, commutators, derivations. 
PROOF.

$$
\begin{aligned}
\Delta_{T^{n}} A & =\sum_{i=0}^{n-1} T^{n-i-1}\left(\Delta_{T} A\right) T^{i} \quad \text { (by induction), } \\
\Delta_{T^{n}} A^{m} & =\sum_{i=0}^{n-1} T^{n-i-1}\left(\Delta_{T} A^{m}\right) T^{i} \\
\Delta_{T} A^{m} & =-\Delta_{A^{m}} T=-\sum_{j=0}^{m-1} A^{m-j-1}\left(\Delta_{T} A\right) A^{j} \\
& =\sum_{j=0}^{m-1} A^{m-j-1}\left(\Delta_{T} A\right) A^{j}
\end{aligned}
$$

Hence

$$
\Delta_{T^{n}} A^{m}=\sum_{i=0}^{n-1} \sum_{j=0}^{m-1} T^{n-i-1} A^{m-j-1}\left(\Delta_{T} A\right) A^{j} T^{i} \text {. Q.E.D. }
$$

IDENTITY II. $\Delta_{T}^{n} A=\sum_{i=0}^{n}(-1)^{i}\left(\begin{array}{c}n \\ i\end{array}\right) T^{n-i} A T^{i}$.

PrOOF. By induction. Q.E.D.

IDENTITY III.

$$
\Delta_{(T-\alpha)^{k}}^{m} A=\sum_{\substack{k_{i}+k_{i}^{\prime}=k-1 ; 1 \leq i \leq m \\ 0 \leq k_{i}, k_{i}^{\prime} \leq k-1}}(T-\alpha)^{k_{1}+k_{2}+\cdots+k_{m}}\left(\Delta_{T}^{m} A\right)(T-\alpha)^{k_{1}^{\prime}+\cdots+k_{m}^{\prime}} .
$$

PROOF. We use induction on $m$.

$$
\begin{aligned}
\Delta_{(T-\alpha)^{k}} A & =\sum_{k_{1}+k_{1}^{\prime}=k-1}(T-\alpha)^{k_{1}}\left(\Delta_{T-\alpha} A\right)(T-\alpha)^{k_{1}^{\prime}} \\
& =\sum_{k_{1}+k_{1}^{\prime}=k-1}(T-\alpha)^{k_{1}}\left(\Delta_{T} A\right)(T-\alpha)^{k_{1}^{\prime}}
\end{aligned}
$$

by Identity I and the fact that $\Delta_{T-\alpha}=\Delta_{T}$ for any scalar $\alpha$. Assume

$$
\begin{aligned}
& \Delta_{(T-\alpha)^{k}}^{m-1} A=\sum_{\substack{k_{i}+k_{i}^{\prime}=k-1 ; \\
1 \leq i \leq m-1}}(T-\alpha)^{k_{1}+k_{2}+\cdots+k_{m-1}}\left(\Delta_{T}^{m-1} A\right)(T-\alpha)^{k_{1}^{\prime}+\cdots+k_{m-1}^{\prime}}, \\
& \Delta_{(T-\alpha)^{k}}^{m} A=\Delta_{(T-\alpha)^{k}}\left[\Delta_{(T-\alpha)^{k}}^{m-1} A\right] \\
& =\Delta_{(T-\alpha)^{k}}\left[\sum_{\substack{k_{i}+k_{i}^{\prime}=k-1 ; \\
1 \leq i \leq m-1}}(T-\alpha)^{k_{1}+k_{2}+\cdots+k_{m-1}}\left(\Delta_{T}^{m-1} A\right)(T-\alpha)^{k_{1}^{\prime}+\cdots+k_{m-1}^{\prime}}\right] \\
& =\sum_{\substack{k_{i}+k_{i}^{\prime}=k-1 ; \\
1 \leq i \leq m-1}}(T-\alpha)^{k_{1}+\cdots+k_{m-1}}\left[\Delta_{(T-\alpha)^{k}}\left(\Delta_{T}^{m-1} A\right)\right](T-\alpha)^{k_{1}^{\prime}+\cdots+k_{m-1}^{\prime}} \\
& =\sum_{\substack{k_{i}+k_{i}^{\prime}=k-1 \\
1 \leq i \leq m}}(T-\alpha)^{k_{1}+\cdots+k_{m}}\left(\Delta_{T}^{m} A\right)(T-\alpha)^{k_{1}^{\prime}+\cdots+k_{m}^{\prime}}
\end{aligned}
$$

by the case $m=1$. Q.E.D. 
IDENTITY IV.

$$
\Delta_{(T-\alpha)^{k}}^{m} A=\left[k(T-\alpha)^{k-1}\right]^{m}\left(\Delta_{T}^{m} A\right)=\left(\Delta_{T}^{m} A\right)\left[k(T-\alpha)^{k-1}\right]^{m}
$$

if $\Delta_{T}^{m+1} A=0(k, m \geq 1)$.

PROOF. $(T-\alpha)^{p}\left(\Delta_{T}^{m} A\right)=\left(\Delta_{T}^{m} A\right)(T-\alpha)^{p}$ for any integer $p \geq 0$ by the hypothesis $\Delta_{T}^{m+1} A=0$. Then Identity IV follows from Identity III. Q.E.D.

IDENTITY V. Let $p$ be any polynomial. If $\Delta_{T}^{m+1} A=0$ for some integer $m \geq 1$, then

$$
\Delta_{p(T)}^{m} A=\left(p^{\prime}(T)\right)^{m}\left(\Delta_{T}^{m} A\right)=\left(\Delta_{T}^{m} A\right)\left(p^{\prime}(T)\right)^{m},
$$

where $p^{\prime}$ is the derivative of $p$ (Identity IV is a special case of Identity V).

PROOF. Note the following facts:

(1) If $T_{1} T_{2}=T_{2} T_{1}$, then $\Delta_{T_{1}} \circ \Delta_{T_{2}}=\Delta_{T_{2}} \circ \Delta_{T_{1}}$ just by the definitions of $\Delta_{T_{1}}$ and $\Delta_{T_{2}}$. Consequently $\Delta_{T_{1}}^{p} \circ \Delta_{T_{2}}^{q}=\Delta_{T_{2}}^{q} \circ \Delta_{T_{1}}^{P}$ for any integers $p, q \geq 1$.

(2) If $T_{1}, T_{2}, \ldots, T_{n}$ are commutative elements, then by induction,

$$
\begin{aligned}
& \Delta_{\alpha_{1} T_{1}+\alpha_{2} T_{2}+\cdots+\alpha_{n} T_{n}}^{m} A=\left(\alpha_{1} \Delta_{T_{1}}+\alpha_{2} \Delta_{T_{2}}+\cdots+\alpha_{n} \Delta_{T_{n}}\right)^{m} A \\
& \quad=\left[\sum_{\substack{i_{i}+i_{2}+\cdots+i_{n}=m \\
0 \leq i_{j} \leq n}} \frac{m !}{i_{1} ! i_{2} ! \cdots i_{n} !} \alpha_{1}^{i_{1}} \alpha_{2}^{i_{2}} \cdots \alpha_{n}^{i_{n}} \Delta_{T_{1}}^{i_{1}} \Delta_{T_{2}}^{i_{2}} \cdots \Delta_{T_{n}}^{i_{n}}\right.
\end{aligned} A
$$

Let $p(T)=\alpha_{1} T^{k_{1}}+\alpha_{2} T^{k_{2}}+\cdots+\alpha_{n} T^{k_{n}}$. Then

$$
\Delta_{p(T)}^{m} A=\left[\sum_{\substack{i_{1}+\cdots+i_{n}=m \\ 0 \leq i_{j} \leq m}} \frac{m !}{i_{1} ! i_{2} ! \cdots i_{n} !} \alpha_{1}^{i_{1}} \alpha_{2}^{i_{2}} \cdots \alpha_{n}^{i_{n}} \Delta_{T^{k_{1}}}^{i_{1}} \Delta_{T^{k_{2}}}^{i_{2}} \cdots \Delta_{T^{k_{n}}}^{i_{n_{n}}}\right] A
$$

by facts (1) and (2). We may assume $k_{i} \geq 1,1 \leq i \leq n$, since $\Delta \beta=0$ for any scalar $\beta$.

$$
\begin{aligned}
& \Delta_{T^{k_{1}}}^{i_{1}} \Delta_{T^{k_{2}}}^{i_{2}} \cdots \Delta_{T^{k_{n}}}^{i_{n}} A=\Delta_{T^{k_{1}}}^{i_{1}} \cdots \Delta_{T^{k_{n-1}}}^{i_{n-1}} \\
& {\left[\sum_{j_{p}(n)+j_{p}^{\prime}(n)=k_{n}-1 ; 1 \leq p \leq i_{n}} T^{j_{1}(n)+\cdots+j_{i_{n}}(n)}\left(\Delta_{T}^{i_{n}} A\right) T^{j_{1}^{\prime}(n)+\cdots+j_{i_{n}}^{\prime}(n)}\right]} \\
& =\sum_{\substack{j_{p}(n)+j_{p}^{\prime}(n)=k_{n}-1 \\
1 \leq p \leq i_{n}}} \cdots \sum_{\substack{j_{p}(1)+j_{p}^{\prime}(1)=k_{1}-1 \\
1 \leq p \leq i_{1}}} T^{r_{1}+\cdots+r_{n}}\left(\Delta_{T}^{i_{1}+\cdots+i_{n}} A\right) T^{r_{1}^{\prime}+\cdots+r_{n}^{\prime}},
\end{aligned}
$$

where $r_{l}=\sum_{t=1}^{i_{l}} j_{t}(l), r_{l}^{\prime}=\sum_{t=1}^{i_{l}} j_{t}^{\prime}(l)$. (Here we repeatedly used Identity III.) Note that

$$
\begin{aligned}
& \sum_{l=1}^{n}\left(r_{l}+r_{l}^{\prime}\right)=i_{1}\left(k_{1}-1\right)+\cdots+ \\
& i_{n}\left(k_{n}-1\right), \\
& i_{1}+i_{2}+\cdots+i_{n}=m, T\left(\Delta_{T}^{m} A\right)=\left(\Delta_{T}^{m} A\right) T .
\end{aligned}
$$

Hence

$$
\begin{aligned}
\Delta_{T^{k_{1}}}^{i_{1}} \cdots \Delta_{T^{k_{n}}}^{i_{n}} A & =k_{1}^{i_{1}} \cdots k_{n}^{i_{n}} T^{i_{1}\left(k_{1}-1\right)} T^{i_{2}\left(k_{2}-1\right)} \cdots T^{i_{n}\left(k_{n}-1\right)}\left(\Delta_{T}^{m} A\right) \\
& =\left[k_{1} T^{k_{1}-1}\right]^{i_{1}}\left[k_{2} T^{k_{2}-1}\right]^{i_{2}} \cdots\left[k_{n} T^{k_{n}-1}\right]^{i_{n}}\left(\Delta_{T}^{m} A\right)
\end{aligned}
$$


Therefore, we have

$$
\begin{aligned}
\Delta_{p(T)}^{m} A & =\left[\sum_{\substack{i_{1}+i_{2}+\cdots+i_{n}=m \\
0 \leq i_{j} \leq m}} \frac{m !}{i_{1} ! i_{2} ! \cdots i_{n} !}\left(\alpha_{1} k_{1} T^{k_{1}-1}\right)^{i_{1}} \cdots\left(\alpha_{n} k_{n} T^{k_{n}-1}\right)^{i_{n}}\right]\left(\Delta_{T}^{m} A\right) \\
& =\left[\alpha_{1} k_{1} T^{k_{1}-1}+\alpha_{2} k_{2} T^{k_{2}-1}+\cdots+\alpha_{n} k_{n} T^{k_{n}-1}\right]^{m}\left(\Delta_{T}^{m} A\right) \\
& =\left[p^{\prime}(T)\right]^{m}\left(\Delta_{T}^{m} A\right)=\left(\Delta_{T}^{m} A\right)\left[p^{\prime}(T)\right]^{m} \text {. Q.E.D. }
\end{aligned}
$$

COROLLARY. The following three conditions are equivalent for any fixed $m \geq 0$.

(i) $\Delta_{T}^{m+1} A=0$;

(ii) $\Delta_{p(T)}^{m+1} A=0$ for each polynomial $p$;

(iii) $\Delta_{S}^{m+1} A=0$ if $S$ is in the norm closed algebra generated by $T$ and $I$.

PROOF. (i) $\Rightarrow$ (ii) by Identity V.

(iii) $\Rightarrow$ (i) is trivial.

(ii) $\Rightarrow$ (iii) by the continuity of the operator $\Delta_{(\cdot)}^{m+1} A$ in the norm topology of $\mathcal{L}(X)$.

LEMMA. If $\exists T \in \mathcal{L}(X)$ having an eigenvalue $\alpha$ such that $X_{T}(\alpha) \neq X$, and $A$ is in the $(m+1)$-commutant of $T$ for some $m \geq 0$, then Lat $A$ is nontrivial.

If, moreover, $\exists \alpha$ such that either $0<\operatorname{dim} X_{T}(\alpha)<\infty$ or $0<\operatorname{dim} X_{T^{*}}(\alpha)<\infty$, then $H$-Lat $A$ is nontrivial.

ProOF. If $m=0$, then $A$ is in the commutant of $T$. This implies that $(T-\alpha)^{n} A=A(T-\alpha)^{n}$ for any integer $n \geq 1$. Hence $\operatorname{ker}(T-\alpha)^{n}(n \geq 1)$ are nontrivial invariant subspaces of $A$ by the hypothesis on $\alpha$.

If $m>0$, by Identity II,

$$
\Delta_{(T-\alpha)^{k}}^{m} A=\sum_{i=1}^{m}(-1)^{i}\left(\begin{array}{c}
m \\
i
\end{array}\right)(T-\alpha)^{k(m-i)} A(T-\alpha)^{k i}+(T-\alpha)^{k m} A
$$

for any integer $k \geq 1$.

On the other hand, by Identity IV, we have $\Delta_{(T-\alpha)^{k}}^{m} A=k^{m}\left(\Delta_{T}^{m} A\right)(T-\alpha)^{(k-1) m}$ for any integer $k \geq 1$ since $A$ is in the $(m+1)$-commutant of $T$.

Comparing the two expressions of $\Delta_{(T-\alpha)^{k}}^{m} A$, we find

$$
(T-\alpha)^{k m} A=k^{m}\left(\Delta_{T}^{m} A\right)(T-\alpha)^{(k-1) m}-\sum_{i=1}^{m}(-1)^{i}\left(\begin{array}{c}
m \\
i
\end{array}\right)(T-\alpha)^{k(m-i)} A(T-\alpha)^{k i}
$$

for any $k \geq 1$.

If $k \geq 2$, then

$$
\begin{aligned}
(T-\alpha)^{k m} A= & {\left[k^{m}\left(\Delta_{T}^{m} A\right)(T-\alpha)^{(k-1)(m-1)}\right.} \\
& \left.\quad-\sum_{i=1}^{m}(-1)^{i}\left(\begin{array}{c}
m \\
i
\end{array}\right)(T-\alpha)^{k(m-i)} A(T-\alpha)^{k i-k+1}\right](T-\alpha)^{k-1} .
\end{aligned}
$$


Therefore, $A\left(\operatorname{ker}(T-\alpha)^{k-1}\right) \subset \operatorname{ker}(T-\alpha)^{k m}$ for any $k \geq 2$; and so

$$
A\left(\bigvee_{n=1}^{\infty} \operatorname{ker}(T-\alpha)^{n}\right) \subset \bigvee_{n=1}^{\infty} \operatorname{ker}(T-\alpha)^{n}
$$

The continuity of $A$ implies that $X_{T}(\alpha)$ is a nontrivial invariant subspace of $A$. If $0<\operatorname{dim} X_{T}(\alpha)<\infty$, then $A\left(X_{T}(\alpha)\right) \subset X_{T}(\alpha)$ implies that $A$ has an eigenvalue $\lambda$. It follows that $\operatorname{ker}(A-\lambda)$ is a nontrivial hyperinvariant subspace of $A$ since $A$ is nonscalar.

Note

$$
\begin{aligned}
\Delta_{T^{*}}^{m} A^{*} & =\sum_{i=0}^{m}(-1)^{i}\left(\begin{array}{c}
m \\
i
\end{array}\right) T^{* m-i} A^{*} T^{* i} \\
& =\left[\sum_{i=0}^{m}(-1)^{i}\left(\begin{array}{c}
m \\
i
\end{array}\right) T^{i} A T^{m-i}\right]^{*}=(-1)^{m}\left(\Delta_{T}^{m} A\right)^{*}
\end{aligned}
$$

So $A$ is in the $(m+1)$-commutant of $T$ iff $A^{*}$ is in the $(m+1)$-commutant of $T^{*}$.

If $0<\operatorname{dim} X_{T^{*}}(\alpha)<\infty$, then $A^{*}\left(X_{T^{*}}(\alpha)\right) \subset X_{T^{*}}(\alpha)$ by the above argument.

Hence $A^{*}$ has an eigenvalue $\mu$ and the closure of the range of $A-\mu$ is a nontrivial hyperinvariant subspace of $A$. Q.E.D.

Now, we prove our theorem.

PROOF OF THE THEOREM. (a) Let $p_{a}(z)=\prod_{j=1}^{l}\left(z-\beta_{j}\right)^{m_{j}}$ be the minimal annihilating polynomial of $T$, where $\beta_{j_{1}} \neq \beta_{j_{2}}$ if $1 \leq j_{1}<j_{2} \leq l$ and $m_{j} \geq 1$ if $1 \leq j \leq l$.

First of all, it is easily seen that $\sigma(T)=\left\{\beta_{1}, \ldots, \beta_{l}\right\}, \sigma_{e}(T)=\left\{\alpha_{1}, \ldots, \alpha_{k}\right\}$, and $l \geq k$. If there is $\beta \in \sigma(T) \backslash \sigma_{e}(T)$, it is well known that $0<\operatorname{dim} X_{T}(\beta)<\infty$. The lemma applies and $H$-Lat $A$ is nontrivial. We may assume that $\sigma(T)=\sigma_{e}(T)$ and $\beta_{i}=\alpha_{i}$ for $1 \leq i \leq k=l$, and so $m_{i} \geq n_{i}$ for $1 \leq i \leq k$.

$p_{a}(T)=0$ implies that every $\alpha_{i}=\beta_{i}$ is an eigenvalue of $T$. For any $1 \leq i_{0} \leq k$, pick a small neighborhood $U$ of $\alpha_{i_{0}}$ such that $U \cap\left(\sigma(T) \backslash\left\{\alpha_{i_{0}}\right\}\right)=\varnothing$.

Define

$$
P_{i_{0}}=\frac{1}{2 \pi i} \int_{\partial U}(z-T)^{-1} d z .
$$

Let $X_{i_{0}}$ be the corresponding subspace of $X$, i.e. $X_{i_{0}}=\overline{P_{i_{0}} X}$. Then $X_{i_{0}}$ is a nontrivial subspace of $X$ by the hypothesis $k \geq 2$. Moreover, $X_{i_{0}}=\{x \in$ $\left.X \mid \lim _{n \rightarrow \infty}\left\|\left(T-\alpha_{i_{0}}\right)^{n} x\right\|^{1 / n}=0\right\}$.

Clearly, $X_{T}\left(\alpha_{i_{0}}\right) \subset X_{i_{0}}$. Hence $\{0\} \neq X_{T}\left(\alpha_{i_{0}}\right) \neq X$. Now the lemma applies and (a) is proved.

(b) By hypothesis, $\exists 0 \neq \alpha \in \sigma(p(T))$. It is well known that $0<\operatorname{dim} X_{p(T)}(\alpha)<$ $\infty$ since $p(T)$ is compact. Since $A$ in the $(m+1)$-commutant of $T$ implies $A$ in the $(m+1)$-commutant of $p(T)$ by the corollary, the conclusion again follows from the lemma.

(c) $0<\operatorname{rank} \Delta_{T}^{m} A<\infty$ is the hypothesis. $A$ in the $(m+1)$-commutant of $T$ implies that $T\left(\Delta_{T}^{m} A\right)=\left(\Delta_{T}^{m} A\right) T$. Then $T$ has an eigenvalue $\alpha$ since $0<$ rank $\Delta_{T}^{m} A<\infty$. Since $k \geq 2$, we may once more use the lemma.

(d) Since $T$ is not algebraic, $p(T)^{N} \neq 0$ for any integer $N \geq 1$. If $m=0$, then $T A=A T$, and so $p(T) A=A p(T)$. Lomonosov's Theorem applies. If $m=1$, then $\Delta_{T}^{2} A=0$. The case $\operatorname{rank} \Delta_{T} A=0$ still follows from Lomonosov's Theorem. Let 
$0<\operatorname{rank} \Delta_{T} A<\infty$. By (b) we may also assume that $\sigma(p(T))=\{0\}$. By the corollary of Identity $\mathrm{V}$, we have $\Delta_{p(T)^{N}}^{2} A=0$ for any integer $N \geq 1$. In particular, $p(T)\left(\Delta_{p(T)} A\right)=\left(\Delta_{p(T)} A\right) p(T)$. Note that $\operatorname{rank}\left(\Delta_{T} A\right)<\infty$ implies rank $\Delta_{p(T)} A<$ $\infty$ for $\Delta_{p(T)} A=p^{\prime}(T)\left(\Delta_{T} A\right)$ by Identity $\mathrm{V}$ (actually rank $\left.\Delta_{p(T)} A \leq \operatorname{rank} \Delta_{T} A\right)$. Let $V$ be the range of $\Delta_{p(T)} A$, then $V$ is a finite-dimensional invariant subspace of $p(T)$. If $V=\{0\}$, i.e. $\Delta_{p(T)} A=0$, then Lomonosov's Theorem applies. Let $V \neq\{0\}$. Since $\sigma(p(T))=\{0\}$ and $\sigma\left(\left.p(T)\right|_{V}\right) \subset \eta(\sigma(p(T))=\sigma(p(T))$ (where $\eta(\sigma(S))$ means the complement of the unbounded component of $\rho(S))$, then $\left.p(T)\right|_{V}$ is quasinilpotent, and so $\left.p(T)\right|_{V}$ is nilpotent since $\operatorname{dim} V<\infty$. Moreover, the order of $\left.p(T)\right|_{V}$ is at most $\operatorname{dim} V$. Hence $\left[\left.p(T)\right|_{V}\right]^{N}=0$ if $N \geq \operatorname{dim} V$.

On the other hand, by Identity IV, it follows that

$$
\Delta_{p(T)^{N+1}} A=(N+1) p^{\prime}(T) p(T)^{N}\left(\Delta_{p(T)} A\right)=0
$$

if $N \geq \operatorname{dim} V$, i.e. $p(T)^{N+1} A=A p(T)^{N+1}$, where $p(T)^{N+1} \neq 0$. Now, we can use Lomonosov's Theorem. (d) is proved.

(e) The case $n_{0}=\operatorname{rank} \Delta_{T} A=0$ is trivial by Lomonosov's Theorem. Let $1 \leq n_{0}=\operatorname{rank} \Delta_{T} A<\infty$. Also we may assume $m=1$. By the hypothesis, $T\left(\Delta_{T} A\right)=\left(\Delta_{T} A\right) T$. Let $W$ be the range of $\Delta_{T} A$; then $W \in$ Lat $T . n_{0}<\infty$ implies that $\sigma\left(\left.T\right|_{W}\right)$ contains only eigenvalues, and also $\sigma\left(\left.T\right|_{W}\right) \subset \eta(\sigma(T))=\sigma(T)$. As in the proof of (a), we may assume $\sigma(T)=\sigma_{e}(T)$.

Let $\sigma\left(\left.T\right|_{W}\right)=\left\{\alpha_{i_{1}}, \ldots, \alpha_{i_{p}}\right\}, i_{1}<i_{2}<\cdots<i_{p}, 1 \leq p \leq k$. If $p=1$, i.e. $\sigma\left(\left.T\right|_{W}\right)$ has only one element, then $\left(\left.T\right|_{W}-\alpha_{i_{1}}\right)^{n_{0}}=0$. Since $m_{0} \geq n_{0},\left(\left.T\right|_{W}-\alpha_{i_{1}}\right)^{m_{0}}=0$. Hence $\operatorname{rank}\left(\left.T\right|_{W}-\alpha_{i_{1}}\right)^{m_{0}-1} \leq 1$. Identity V implies

$$
\begin{aligned}
\Delta_{q(T)} A & =q^{\prime}(T)\left(\Delta_{T} A\right)=\left[\sum_{i=1}^{k} m_{0}\left(T-\alpha_{i}\right)^{m_{0}-1} \prod_{j \neq i}\left(T-\alpha_{j}\right)^{m_{0}}\right]\left(\Delta_{T} A\right) \\
& =m_{0}\left[\prod_{j \neq i_{1}}\left(T-\alpha_{j}\right)^{m_{0}}\right]\left(T-\alpha_{i_{1}}\right)^{m_{0}-1}\left(\Delta_{T} A\right)
\end{aligned}
$$

since $\left(\left.T\right|_{W}-\alpha_{i_{1}}\right)^{m_{0}}=0$ implies $\left(T-\alpha_{i_{1}}\right)^{m_{0}}\left(\Delta_{T} A\right)=0 . \operatorname{Rank}\left(\left.T\right|_{W}-\alpha_{i_{1}}\right)^{m_{0}-1} \leq 1$ implies $\operatorname{rank}\left(T-\alpha_{i_{1}}\right)^{m_{0}-1}\left(\Delta_{T} A\right) \leq 1$. Therefore

$$
\operatorname{rank}\left(\Delta_{q(T)} A\right)=\operatorname{rank}(q(T) A-A q(T)) \leq 1 .
$$

Note that $m_{0} \geq n_{i}$ for each $1 \leq i \leq k$, and so $q(T)$ is compact. Now we can use Theorem 2 in [4]; $H$-Lat $A$ is nontrivial.

If $1<p \leq k$, first note that the eigenvectors for distinct eigenvalues of $\left.T\right|_{W}$ are linearly independent. $\operatorname{dim} W=n_{0}$ implies that $\left(\left.T\right|_{W}-\alpha_{i_{l}}\right)^{n_{0}-1}=0$ for each $1 \leq l \leq p$. Hence $\left(\left.T\right|_{W}-\alpha_{i_{l}}\right)^{m_{0}-1}=0,1 \leq l \leq p$. This implies that $\left(T-\alpha_{i_{l}}\right)^{m_{0}-1}\left(\Delta_{T} A\right)=0$ for each $1 \leq l \leq p$.

$$
\Delta_{q(T)} A=\left[\sum_{i=1}^{k} m_{0}\left(T-\alpha_{i}\right)^{m_{0}-1} \prod_{j \neq i}\left(T-\alpha_{j}\right)^{m_{0}}\right]\left(\Delta_{T} A\right)=0
$$

since each summand in the above sum has a factor $\prod_{l=1}^{p}\left(T-\alpha_{i_{l}}\right)^{m_{0}-1}$ which kills $\left(\Delta_{T} A\right)$. Hence $\Delta_{q(T)} A=q(T) A-A q(T)=0$. Since $q(T) \neq 0$ is compact, the conclusion holds by Lomonosov's Theorem. (e) is proved. Q.E.D. 
REMARKS AND QUESTIONS. 1. In conclusion (a), $k>1$ is essential. In [5], it was pointed out that there always is a nilpotent element $T$ of order 2 such that $A$ is in the 2-commutant of $T$.

2. Concerning (c), we have the following question: Can we remove the hypothesis $\operatorname{rank} \Delta_{T}^{m} A<\infty$ ? If $\operatorname{ker}(p(T)) \neq\{0\}$, then $T$ has an eigenvalue. As in the proof of (a), we can prove that Lat $A$ is nontrivial without the assumption $\operatorname{rank} \Delta_{T}^{m} A<\infty$. What about the case that $T$ is not algebraic and $\operatorname{ker}(p(T))=\{0\}$ ? For this case, can we expect that $H$-Lat $A$ is nontrivial? Also if we keep the condition $\operatorname{rank} \Delta_{T}^{m} A<\infty$, can we remove the condition $k>1$ ? For the case $m \leq 1$, (d) says "yes."

3. If $\exists m \geq 0$ and a nonzero compact operator $T$ such that $\Delta_{T}^{m} A$ is a rank one operator, what can we say about the (hyper)invariant subspace of $A$ ? For $m=0$, see [4].

4. Combining (b) and (e) above we obtain an improvement of Theorem 2 in [5]. ACKNOWLEDGMENT. The author wishes to thank the referee and Professor L. Brown for reading this paper and giving him some corrections and advice.

\section{REFERENCES}

1. S. W. Brown, Connections between an operator and a compact operator that yield hyperinvariant subspaces, J. Operator Theory 1 (1979), 117-121.

2. J. Daughtry, An invariant subspace theorem, Proc. Amer. Math. Soc. 49 (1975), 267-269.

3. D. A. Herrero, Approximation of Hilbert space operators, vol. 1, Pitman, Boston, London, Melbourne, 1982.

4. H. W. Kim, C. M. Pearcy, and A. L. Shields, Rank-one commutators and hyperinvariant subspaces, Michigan Math. J. 22 (1975), 193-194.

5. H. Lin, Small commutators and hyperinvariant subspaces, Proc. Amer. Math. Soc. 96 (1986), 443-446.

6. V. Lomonosov, Invariant subspace for operators commuting with compact operators, Funktsional Anal. i Prilozhen. 7 (1973), 55-56. (Russian)

7. H. Radjavi and P. Rosenthal, Invariant subspaces, Springer-Verlag, Berlin and New York, 1973, p. 8.

Department of Mathematics, Purdue University, West Lafayette, Indiana 47907 